\title{
Corrigendum
}

In the paper by Xu et al., Plant Molecular Biology Volume 58, pp. 773-784, the affiliation was incomplete: The first two authors contributed equally to this paper. Please find the correct affiliation below:

\section{Activation of the $W U S$ gene induces ectopic initiation of floral meristems on mature stem surface in Arabidopsis thaliana}

Yun-Yuan $\mathrm{Xu}^{1, \dagger}$, Xiao-Min Wang ${ }^{1, \dagger}$, Jia $\mathrm{Li}^{2}$, Ju-Hua $\mathrm{Li}^{1}$, Jin-Song $\mathrm{Wu}^{1}$, John C. Walker ${ }^{3}$, Zhi-Hong $\mathrm{Xu}^{1}$ and Kang Chong ${ }^{1, *}$

${ }^{1}$ Key Laboratory of Photosynthesis \& Molecular Physiology, Institute of Botany, Chinese Academy of Sciences, Nanxincun 20, XiangShan, Beijing 100093, China (*author for correspondence; e-mail chongk@ibcas.ac.cn); ${ }^{2}$ Department of Botany and Microbiology, University of Oklahoma, Norman, OK 73019-6131, USA; ${ }^{3}$ Division of Biological Sciences, University of Missouri, Columbia, MO 65211, USA;

${ }^{\dagger}$ These authors contributed equally to this work

Figure 3 contained several errors. The correct panels (A) and (B) are depicted below as well as the current incorrect version.

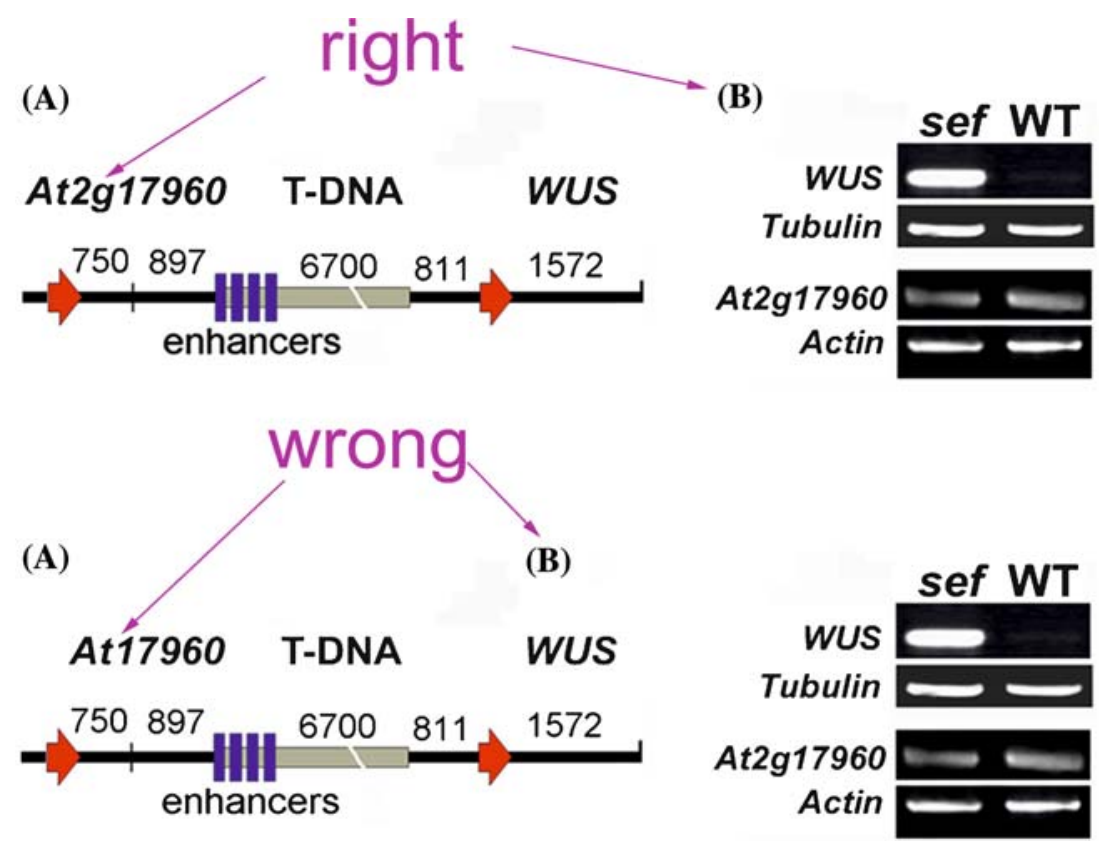

